\title{
Vergesellschaftung von Fürsorgearbeit - Erfahrungen aus der Altenbetreuung in Schweden
}

Wie sollen ältere Menschen versorgt werden? Welche Rolle sollen dabei der Staat, der Markt, die Familie oder der Dritte Sektor übernehmen? Was bedeuten die Strukturentscheidungen für Ungleichheiten zwischen den Geschlechtern oder verschiedenen Gruppen von Frauen? Die Antworten auf diese Fragen unterscheiden sich erheblich zwischen den Wohlfahrtsstaaten. Schweden gilt als Prototyp eines Wohlfahrtsstaats, der die Etablierung von Fürsorgearbeit als bezahlte Erwerbsarbeit unter Verantwortung des Staates forciert. Welche Bedeutung dies für die zumeist weiblichen Pflegenden gewinnt und welche Chancen und Schwierigkeiten beim Aufbau eines Arbeitsmarktsegments im Zeitverlauf erkennbar werden, steht im Mittelpunkt des Beitrags.

\section{Problemstellung}

Die Gleichstellung von Frauen und Männern auf dem Arbeitsmarkt hat zwei grundlegende Prämissen: Dies sind die $I n$ tegration von Frauen in den Arbeitsmarkt und die Qualität der Arbeitsmarktintegration, d.h. es geht um Fragen der Lohn- und Arbeitssituation von Frauen sowie um die entstehenden Muster von Geschlechtersegregation. Die Erwerbsintegration wird häufig aus der Perspektive der Vereinbarkeit von Beruf und Familie diskutiert, weniger beachtet wird hingegen die Frage, inwieweit Frauen prinzipiell Möglichkeiten zur Erwerbsarbeit offenstehen.

Im Fokus dieses Beitrages stehen zunächst die Möglichkeiten der Erwerbsintegration unterschiedlicher Gruppen von Frauen durch die Verberuflichung des traditionell weiblichen Tätigkeitsfelds „der Versorgung älterer Menschen " im deutschschwedischen Vergleich. Daran anschlieBend wird der Aufbau dieses Arbeitsmarktsegments in Schweden seit Ende der 1950er Jahre betrachtet. In Schweden hat sich die Altenbetreuung mittlerweile als ein umfassendes und reguläres Tätigkeitsfeld auf dem Arbeitsmarkt etabliert. Im Gegensatz dazu umfasst das Tätigkeitsfeld in Deutschland ein weites Spektrum unterschiedlicher Arbeitsverhältnisse, angefangen von regulären, auf dem Arbeitsmarkt angesiedelten Beschäftigungsverhältnissen über Tätigkeiten, die auf einem grauen Markt hergestellt werden bis hin zu symbolisch bezahlter Familienarbeit. Um grundlegende Analysedimensionen für den Ländervergleich aufzeigen zu können, werden zunächst zentrale Diskussionsstränge der international vergleichenden Wohlfahrtsstaatsforschung vorgestellt.

\section{Verberuflichung der
Versorgung älterer Menschen}

\subsection{WISSENSCHAFTLICHE DEBATTE}

Inspiriert durch Esping-Andersens Ansatz zu Wohlfahrtsstaatsregimen (1990) und die sich daran aus einer Geschlechterperspektive entzündende Kritik entstand eine intensive wissenschaftliche Debatte zur Verortung von „social care“, d.h. der Fürsorgearbeit für Kinder und ältere Menschen in wohlfahrtsstaatsstaatlichen Arrangements, und über deren zentrale Dimensionen im Ländervergleich (Anttonen/ Sipilä 1996; Knijn/Kremer 1997; Bettio/ Plantenga 2004). Das Konzept „social care“ verbindet private, familiäre Versorgung mit Versorgungsaktivitäten, die in öffentlichen Sektoren angesiedelt sind, und es verknüpft somit zumeist getrennt analysierte gesellschaftliche Bereiche. Auf dieser Basis können die komplexe gesellschaftliche Verortung von Versorgung und deren Konsequenzen gerade für die gesellschaftliche Position von Frauen aufgezeigt werden. Als eine entscheidende Dimension erweist sich dabei die im Ländervergleich unterschiedliche Bedeutung von Wohlfahrtsstaatssektoren - Staat, Markt, Familie und dritter Sektor - und deren Zusammenspiel (z. B. Welfare Mix Ansätze: Evers/Wintersberger 1990; Evers/Svetlik 1993). Unter dem Schlagwort "Care goes public" wurde der
Prozess der zunehmenden Verortung der traditionell familiären Fürsorgearbeit in öffentlichen Sektoren der Gesellschaft diskutiert (Anttonen et al. 2003).

Neuere Entwicklungen in den Pflegepolitiken, als ein zentraler Politikbereich der Fürsorgearbeit, erbrachten immer komplexere Beziehungen zwischen den Sektoren, wobei insbesondere zwischen Finanzierung und Ausführung der Tätigkeit unterschieden wird. Der Staat tritt vermehrt als Finanzier und weniger als Lieferant von Pflegetätigkeiten auf. Er unterstützt beispielsweise auf der Basis von Geldleistungen die familiäre Pflege oder finanziert die auf dem Markt bzw. im Dritten Sektor hergestellten Aktivitäten. Im Hintergrund stehen länderspezifische Normen zur Rolle und Verantwortung der Familie, des Staates, des Marktes und des Dritten Sektors für die Pflegetätigkeit. Die immer komplexer werdenden Verbindungen zwischen den einzelnen Sektoren erforderten auch eine Weiterentwicklung des Konzepts „social care“. In ihrem Ansatz definierten Daly/Lewis (1998) drei Dimensionen zur Analyse von „social care“: die Ver-

\footnotetext{
Hildegard Theobald, Dr., Professorin für Organisationelle Gerontologie am Zentrum Altern und Gesellschaft der Hochschule Vechta - Universität. Arbeitsschwerpunkte: International vergleichende Wohlfahrtsstaatsforschung, insbesondere Social Care Systeme, Arbeitsmarkt und Professionalisierung, soziale Ungleichheit im Zusammenspiel von Geschlecht und sozio-ökonomischer Klasse. e-mail. hildegard.theobald@uni-vechta.de
} 
teilung der Pflegearbeit und der Kosten zwischen den einzelnen Sektoren sowie deren normative Basis.

Für Schweden konnte Evertsson (2000) die Bedeutung des Wohlfahrtsstaats für die Entwicklung der frauendominierten Gesundheits- und Pflegeberufe aufzeigen. Er führt aus, dass der Wohlfahrtsstaat in Schweden einen stabilen Arbeitsmarkt für Pflegetätigkeiten geschaffen hat, der eine gute Ausgangsbasis für eine Professionalisierung der Pflegetätigkeiten bot. Schweden gilt in der international vergleichenden Wohlfahrtsstaatsforschung als ein Prototyp des sozialdemokratischen Modells, charakterisiert durch eine Expansion von sozialen Dienstleistungen unter staatlicher Regie trotz einer Öffnung für Marktmechanismen seit dem Beginn der 1990er Jahre. Deutschland hingegen gilt als ein Repräsentant eines familienorientierten Modells mit der Betonung der Versorgung im familiären Rahmen, basierend auf Normen familialer Solidarität, wobei seit den 1990er Jahren familiäre Pflegearbeit auf der Basis von Geldleistungen vermehrt direkt unterstützt wird. Bettio/Plantenga (2004) ordnen Deutschland daher in ihrem Vergleich einer Gruppe von Ländern zu, die die Versorgung im familiären Rahmen jedoch mit staatlicher Unterstützung vorsehen.

\subsection{ARBEITSMARKT- UND GLEICHSTELLUNGSPOLITISCHE DIMENSIONEN}

Gerade aufgrund der weiblichen Konnotation von Fürsorgearbeit - ob bezahlt oder unbezahlt - beeinflusst die wohlfahrtsstaatliche Entwicklung die Verortung von Frauen in den gesellschaftlichen Bereichen Markt, Staat, Familie und Dritter Sektor. Dies spiegelt sich in Höhe und Form weiblicher Erwerbstätigkeit und der Geschlechtersegregation auf dem Arbeitsmarkt wider. Eine Analyse der Erwerbsbeteiligung von Frauen im Ländervergleich ergab, dass insbesondere der Ausbau der Kinder- und Altenbetreuung als bezahlte Erwerbsarbeit eine entscheidende Rolle für die Höhe und Form weiblicher Erwerbsbeteiligung spielt (Anttonen/Sipilä 1996). Eine differenzierte Betrachtung der weiblichen Erwerbstätigen in Abhängigkeit von ihrem Qualifikationsniveau erbrachte für Schweden, dass insbesondere Frauen mit einer einfachen oder ohne formale Qualifikation durch die Expansion der Altenbetreuung einen $\mathrm{Zu}$ - gang zum Arbeitsmarkt finden (Szebehely 1998).

Ein detaillierter Vergleich der Arbeitsmarktbeteiligung deutscher und schwedischer Frauen mit einem unterschiedlichen Qualifikationshintergrund kann diesen Zusammenhang erhellen (Theobald 1999; Theobald/Maier 2002). Im Ländervergleich zeigt sich, dass sich die höhere Erwerbsquote schwedischer Frauen insbesondere auf eine höhere Erwerbsbeteiligung von Frauen mit einer mittleren, einfachen bzw. ohne formale Qualifikation zurückführen lässt. Dies sind Frauen, die in Schweden - im Gegensatz zu Deutschland - zu einem großen Anteil über die sozialen Dienstleistungen wie Kinder- oder Altenbetreuung in den Arbeitsmarkt integriert werden.

Neuere Statistiken bestätigen die Bedeutung gerade der Altenbetreuung für die Erwerbsbeteiligung von Frauen. In Schweden waren 2005 in der kommunalen Altenbetreuung - ambulant und stationär $7,39 \%$ aller Erwerbstätigen und 12,7 \% der weiblichen Erwerbstätigen beschäftigt. In Deutschland hingegen waren im Rahmen der Pflegeversicherung in der ambulanten und stationären Versorgung lediglich 2,08 \% aller Erwerbstätigen und 3,97 \% aller weiblichen Beschäftigten tätig. In beiden Ländern zeigt sich eine ausgeprägte Frauendominanz mit einem Frauenanteil von ca. 90 \% in Schweden und ca. $86 \%$ in Deutschland. Der Vergleich der nationalen Statistiken weist zwar Probleme auf, die jedoch das Ergebnis nicht grundlegend verändern. ${ }^{1}$ Nach wie vor bietet der Sektor in Schweden Frauen mit einer einfachen oder ohne formale Qualifikation einen Zugang zum Arbeitsmarkt (Abschnitt 3).

Die Formen der Vergesellschaftung von Fürsorgearbeit in der Altenbetreuung beeinflussen daher nicht nur die Gleichheit zwischen den Geschlechtern - z. B. in der Frage der Arbeitsmarktbeteiligung -, sondern auch die Gleichheit zwischen verschiedenen Gruppen von Frauen in einer Klassenperspektive. Neuere Entwicklungen weisen noch Ethnizität als eine dritte zentrale Dimension von Ungleichheit in dem Tätigkeitsfeld aus (Graham 1991; Hochschild 2000). Italien als Repräsentant eines familienorientierten Modells, das nach Bettio/Plantenga (2004) die Verantwortung für die Versorgung noch weitgehend an die Familie delegiert, zeigt, wie die zumeist illegale Beschäftigung von Migrantinnen in der häuslichen Versorgung älterer Men- schen als eine Lösung des Care-Dilemmas angesehen werden kann. Ein Care-Dilemma, das entsteht, wenn ausgehend von der Norm familiärer Verantwortlichkeit unter veränderten sozio-ökonomischen Bedingungen ein nur begrenztes Angebot an Dienstleistungen vorhanden ist (Lamura 2007).

Schweden gilt im Gegensatz zu Italien als Repräsentant eines Wohlfahrtsstaats, in dem der Staat schon früh Verantwortung für die Versorgung älterer Menschen übernommen hat und unter öffentlicher Regie ein Tätigkeitsfeld auf dem Arbeitsmarkt aufgebaut wurde. Dies erhöht, wie bereits angesprochen, die Zugangschancen bestimmter Gruppen von Frauen zum Arbeitsmarkt. $\mathrm{Zu}$ untersuchen bleibt jedoch die Qualität der entstandenen Tätigkeiten. Zentrale Dimensionen dafür sind die Arbeitssituation der Pflegekräfte, Anstellungsformen sowie Möglichkeiten von Qualifikations- und Kompetenzentwicklung. Zudem muss berücksichtigt werden, dass sich die "Versorgung älterer Menschen" erst in einem langwierigen Prozess als Erwerbsarbeit etabliert hat, der eng mit der Entwicklung der Altenbetreuung in verschiedenen Zeitperioden verknüpft ist. Deswegen werden in der folgenden Analyse zwei Zeitperioden mit sehr unterschiedlichem Einfluss differenziert.

\footnotetext{
Aufgrund von Abgrenzungsproblemen gibt es keine wirklich vergleichbaren Statistiken für beide Länder. Dies betrifft zum einen die Unterscheidung zwischen Grundpflege und Behandlungspflege sowie zum anderen die Versorgungsangebote auf kommunaler Ebene. In beiden Ländern sind in die Statistiken Beschäftigte in der Grundpflege, aber auch zum Teil in der Behandlungspflege eingeschlossen. In Deutschland erbringen $94 \%$ der Dienstleister in der Grundpflege - bezahlt nach dem Pflegeversicherungsgesetz - auch Leistungen in der ambulanten Behandlungspflege (Krankenversicherungsgesetz), wobei das Personal in beiden Bereichen eingesetzt werden kann. In Schweden haben ca. 50 \% der Gemeinden die ambulante Behandlungspflege übernommen und einen gemeinsamen Pflegedienst aufgebaut. Des Weiteren bieten die schwedischen Gemeinden auch soziale Dienste an, wie z. B. Transport- oder Begleitdienste, die bei uns auch von den Gemeinden angeboten, aber damit nicht von der Pflegestatistik erfasst werden. Allerdings sind dies bei uns häufig Einsatzbereiche von Zivildienstleistenden, ehrenamtlich Tätigen oder auch Beschäftigten im Rahmen von Arbeitsmarktprogrammen.
} 


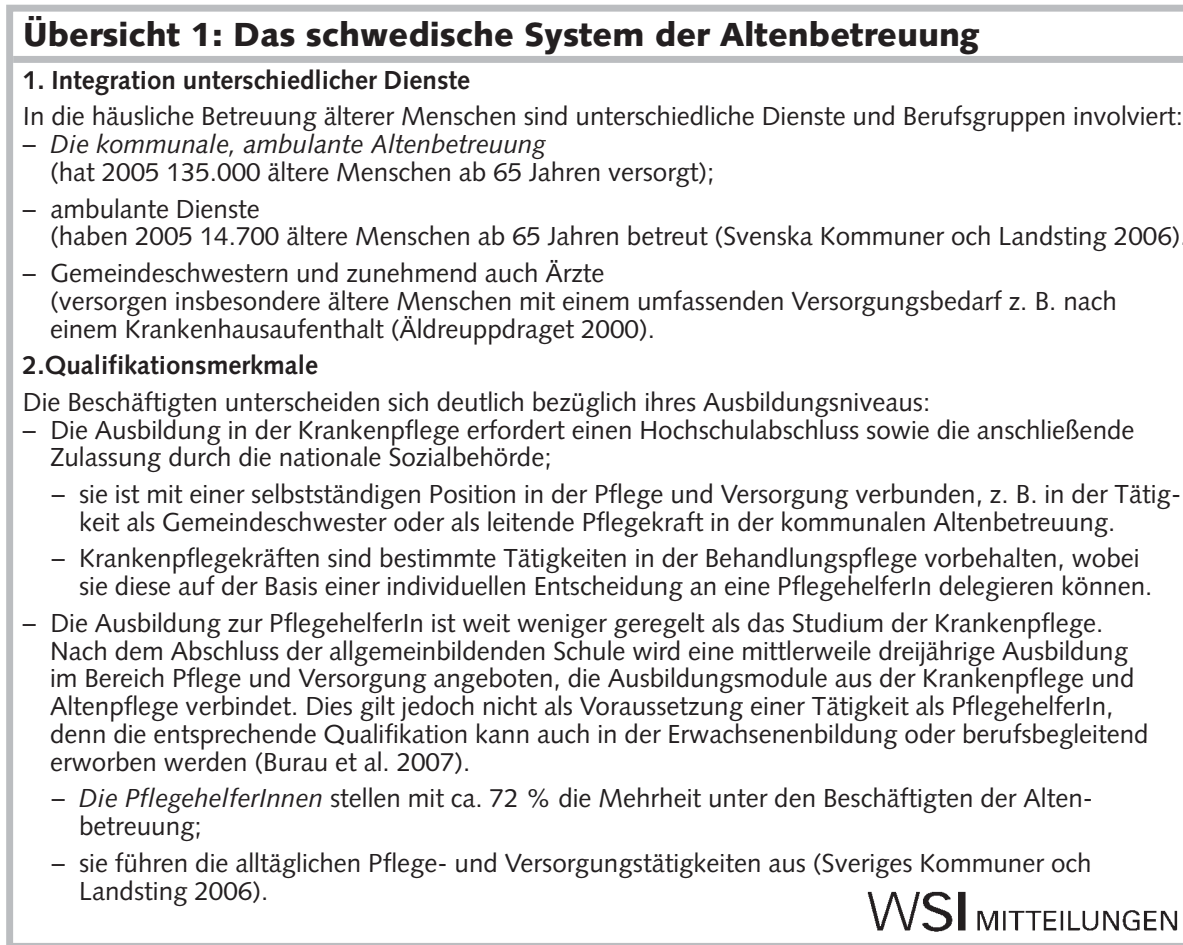

\section{2 \\ Altenbetreuung in Schweden \\ 3.1 VERGESELLSCHAFTUNG DER ALTENBETREUUNG: 1950-1990}

Im Schweden begann der Aufbau sozialer Dienstleistungen für ältere Menschen schon im Verlauf der 1950er Jahre und avancierte schnell $\mathrm{zu}$ einem zentralen Element des universellen Wohlfahrtsstaats. Bis zu diesem Zeitpunkt war die Beschäftigung von bezahlten Kräften in privaten Haushalten zur Unterstützung von Hausund Betreuungsarbeit typisch für den (groß)bürgerlichen Haushalt und wurde privat finanziert. Allerdings wurde die frühe Institutionalisierung der Betreuung älterer, versorgungsbedürftiger Menschen zunehmend als "gesellschaftliche Misere“ wahrgenommen und entzündete eine Debatte über Ansätze häuslicher Versorgung. Diskutiert wurde, wer Zugang zu Versorgungsleistungen erhalten sollte, wie diese finanziert werden sollten und wer sie ausführen sollte. Schließlich konnte sich die Zielsetzung durchsetzen, den Zugang zu sozialen Dienstleistungen als ein soziales Recht zu definieren, das nach dem Bedürfnisprinzip und nicht nach der Einkommenssituation gewährleistet werden sollte. Soziale Dienstleistungen sollten auf der
Basis von Steuerleistungen finanziert und von den Kommunen angeboten werden (Gough 1994). Einen weiteren Impuls erhielt diese Politikausrichtung mit der seit den 1960er Jahren formulierten Zielsetzung, Frauen in den Arbeitsmarkt zu integrieren. Der Ausbau sozialer Dienstleistungen wurde als eine grundlegende Voraussetzung für die Erwerbsbeteiligung von Frauen betrachtet (Hirdman 1990).

Die öffentliche Finanzierung und Organisation sozialer Dienste in der häuslichen Versorgung bildeten den Ausgangspunkt für eine zunehmende staatliche Regulierung von Arbeitsaufgaben, Qualifikationsbedarf und -anforderungen und für die Entwicklung eines Berufsfelds (Gough 1994; Johansson 1997; Szebehely 1995, 1996). Als Barrieren für die Etablierung der Tätigkeit als Erwerbsarbeit erwiesen sich: ihre Tradition als Hausarbeit in bürgerlichen Familien, das Angebot von gering entlohnten Betreuungsleistungen auf einem grauen Markt, die unbezahlte Betreuungsarbeit von „Hausfrauen" in der Familie und die Anfänge sozialer Betreuungsarbeit als ehrenamtliche Tätigkeit zur Unterstützung „Bedürftiger“. Vor diesem Hintergrund wurde die häusliche Betreuung nicht als qualifizierte Tätigkeit wahrgenommen, sondern eher als eine symbolisch zu bezahlende Aufgabe, deren Ausführung durch abstrakte Prinzipien und bürokratische Regulierungen eher behindert wird
(Gough 1994; Johannsen 1997; Johanssen/ Noren 2001).

Bis in die 1980er Jahre dominierten Haushaltsdienstleistungen die Arbeitsaufgaben in der häuslichen Betreuung mit entsprechend niedrigen Qualifikationsanforderungen (zum Folgenden: Szebehely 1995). Eingestellt wurden zumeist Frauen ohne formale Qualifikation, die nach einer Familienphase in den Arbeitsmarkt zurückkehrten. Erst im Verlauf der 1980er Jahre wurde unter dem Schlagwort „Hilfe zur Selbsthilfe" die traditionelle Hausarbeit durch aktivierende, präventive und rehabilitative Pflegeaufgaben ergänzt. Parallel zu der Erweiterung der Arbeitsaufgaben wurden die Arbeitsbedingungen neu definiert, womit die Verberuflichung der Tätigkeit gefördert wurde. Dazu gehörten die Betonung der Qualifikation mit der entsprechenden Entwicklung eines Ausbildungscurriculums und eine mehr berufsförmig organisierte Arbeitszeit, die zunehmend unbefristete Arbeitsverträge mit einer zumindest 30-stündigen Wochenarbeitszeit vorsah. Die neuen Arbeitsbedingungen sollten die Tätigkeit auch für die besser ausgebildete, jüngere Generation attraktiv gestalten, da die vormals in der Familie tätigen Frauen bis Ende der 1970er Jahre weitgehend in den Arbeitsmarkt integriert waren. Ähnlich wie für die Sozialarbeit und die Krankenpflege wurden neben einer Berufsausbildung zudem Hochschulstudiengänge im Bereich „Altenbetreuung" eingerichtet. Bis Ende der 1980er Jahre entwickelte sich ein neues, hierarchisch organisiertes Berufsfeld mit einem Anteil von ca. $10 \%$ von (zumeist weiblichen) Hochschulabsolventen (aus der Krankenpflege bzw. Altenbetreuung) unter den Beschäftigten, die in der Regel in leitenden Positionen tätig waren. Die alltägliche Pflegearbeit wurde von Pflegehelferinnen und -helfern mit und ohne formale Qualifikation durchgeführt, deren Lohnniveau dem Niveau gleichqualifizierter Frauen in anderen Arbeitsmarktbereichen entsprach (eigene Berechnungen auf der Grundlage von SCB 1990; 1993; vgl. auch Übersicht 1).

\subsection{RESTRUKTURIERUNG UND NEUENTWICKLUNG: 1990 BIS HEUTE}

\section{GEGENLÄUFIGE ENTWICKLUNGEN}

Seit den 1990er Jahren werden zwei unterschiedliche Entwicklungen erkennbar: In einer Situation ökonomischer Restriktio- 
nen dominierten bis in die zweite Hälfte der 1990er Jahre hinein Anstrengungen zur Restrukturierung und zum Abbau von Personal sowie zur Reorganisation der Versorgung. Dagegen kann seit Ende der 1990er Jahre wieder eine gegenläufige Entwicklung mit vermehrten Einstellungen und einer Erhöhung ökonomischer Ressourcen beobachtet werden. Beide Entwicklungen haben jeweils erhebliche Konsequenzen für die Verberuflichung der Tätigkeit und die berufliche Situation der zumeist weiblichen Beschäftigten.

Zu Beginn der 1990er Jahren konnten sich zunehmend ökonomische Argumente, wie beispielsweise das der Kostenersparnis, auf politischer Ebene durchsetzen (Äldreuppdraget 1996, 2000). Die Grundprinzipien der Versorgung älterer Menschen blieben dabei unverändert (Szebehely 2000): Die Dienste sollten nach wie vor steuerfinanziert werden sowie nach Bedarf, und nicht nach Kaufkraft, zugänglich sein. Ältere Menschen sollten auch weiterhin Zugang zu einer angemessenen und guten Versorgung erhalten - allerdings unter veränderten Organisationsformen. Infragegestellt wurde die Rolle der Kommunen als Lieferant - nicht als Finanzier - von Dienstleistungen. Markt, Freiwilligensektor und informelle Pflege sollten aufgewertet werden. Die Zulassung von nicht-öffentlichen Anbietern und damit von Konkurrenz sollte die Arbeitsweise effektiver gestalten, Kosten sparen und den Nutzern mehr Wahlmöglichkeiten geben. Nationale Gesetze boten die Möglichkeit, unterschiedliche Anbieter zuzulassen. Dennoch blieb es den Kommunen überlassen, inwieweit sie tatsächlich weitere, nicht-öffentliche Anbieter akzeptieren wollten, was zu großen Unterschieden zwischen den Kommunen führte (Brodin 2005). Im Jahr 2005 wurden $10 \%$ der ambulant versorgten älteren Menschen und $13 \%$ der in stationären oder betreuten Wohnformen lebenden Älteren von nicht-kommunalen zumeist privatwirtschaftlich organisierten Dienstleistern - versorgt (Sveriges Kommuner och Landsting 2006). Eine Untersuchung der nationalen Sozialbehörde aus dem Jahr 2003 ergab allerdings, dass in den Kommunen, die die Altenbetreuung für konkurrierende Anbieter geöffnet hatten, die Kosten stärker als in den anderen Kommunen angestiegen waren (SOS 2004).

\section{PERSONALABBAU}

Die neuen politischen Richtlinien und insbesondere die ökonomischen Restriktionen auf kommunaler Ebene führten zu Personalabbau und einer Restrukturierung unter den Beschäftigten. Im Vergleich zu den weiteren Wohlfahrtsstaatssegmenten, wie beispielsweise Kinderbetreuung oder Gesundheitswesen, fielen in der Altenbetreuung die Personalreduktionen moderat aus. Die Bedeutung der Veränderungen in der Altenbetreuung kann jedoch nicht ohne die Berücksichtigung der drastischen Personalkürzungen im Gesundheitswesen bewertet werden. Denn die Personalkürzungen im Gesundheitswesen wurden im Zusammenhang mit Kürzungen der Verweildauer in Krankenhäusern durchgeführt, womit sich ein erhöhter Bedarf an ambulanter Versorgung ergab. Unter derart veränderten Voraussetzungen in der Versorgung und angesichts der zunehmenden Alterung der Bevölkerung führte folglich auch der nur moderate Personalabbau zu einer deutlich restriktiveren Verteilung von Dienstleistungen und zu erheblichen Konsequenzen für die Beschäftigten (Szebehely 2000).

Überdurchschnittlich betroffen von den Kürzungen waren Beschäftigte ohne formale Qualifikation, während sich der Anteil der Pflegekräfte mit einem Hochschulabschluss oder einer formalen Berufsausbildung erhöhte. Die Veränderungen wurden teilweise durch Frühpensionierungen der insbesondere älteren Frauen ohne formale Qualifikation erreicht sowie durch verstärkte Anstrengungen, die verbleibenden Pflegekräfte zu qualifizieren. Die Vorstellung setzte sich durch, dass im Zuge von Personalkürzungen nur dann eine adäquate Versorgung aufrechterhalten werden kann, wenn gleichzeitig die Qualifikation der Beschäftigten steigt (Gonäs et al. 1997). Die höheren Qualifikationsanforderungen erschienen auch notwendig vor dem Hintergrund der Restrukturierungen im Gesundheitswesen, denn die vermehrte ambulante Versorgung Kranker erforderte zumindest grundlegende pflegerische Kenntnisse (Johannson 1997).

\section{ARBEITSBELASTUNGEN}

Können die Veränderungen bezüglich der Qualifikationsanforderungen noch widersprüchlich bewertet werden - sie bewirkten einen erschwerten Zugang von Pflegekräf- ten ohne formale Qualifikation in den Arbeitsbereich bei einer gleichzeitigen Verbesserung des Zugangs zu Ausbildungen -, weisen andere Indikatoren bezüglich der Arbeitssituation eindeutig negative Konnotationen auf. Untersuchungen zu Arbeitsbelastung und Arbeitssituation zeigen für den Verlauf der 1990er Jahre eine deutliche Zunahme von psychischen und physischen Belastungen, was mit den personellen Veränderungen und Kürzungen erklärt werden kann (Bäckmann 2001). Während im Jahr 1991 lediglich 14 \% der unmittelbar mit der Pflege beschäftigten Kräfte angaben, zuviel zu tun zu haben, stieg ihr Anteil auf $39 \%$ im Jahr 2000. Dabei klagten Befragte besonders über physische und psychische Müdigkeit und Erschöpfung (Gustafsson/Szebehely 2005). Zudem konstatierten sie, dass die Versorgung der Klientel nicht mehr ausreichend gewährleistet werden könnte.

\section{BESCHÄFTIGUNGSFORMEN}

Die Restrukturierungen beeinflussten auch die Anstellungsformen in der Altenbetreuung. War die Entwicklung in den 1980er Jahren durch einen erhöhten Anteil von Festeinstellungen gekennzeichnet, so zeigt sich in den 1990er Jahren eine gegenläufige Tendenz. Der Anteil der lediglich stundenweise beschäftigten Pflegehelferinnen und -helfer mit einer ökonomisch unsicheren Situation hat sich zwischen 1990 und 1997 verdoppelt (Szebehely 2000). In einer Befragung im Jahr 2003 gaben $72 \%$ der befragten Pflegekräfte an, einen unbefristeten Arbeitsvertrag zu besitzen, $9 \%$ einen Zeitvertrag und $19 \%$ wurden stundenweise beschäftigt, was in etwa den statistischen Angaben auf kommunaler Ebene entspricht. Weiterhin waren lediglich $28 \%$ der Pflegehelferinnen und -helfer vollzeittätig. Diese Angaben spiegeln oft unerwünschte Anstellungsformen. So gaben in der Befragung $26 \%$ der teilzeittätigen Pflegekräfte an, eine Vollzeittätigkeit anzustreben, $83 \%$ der zeitlich befristeten und $37 \%$ der stundenweise Beschäftigten wünschten sich einen unbefristeten Arbeitsvertrag (Gustaffsson/Szebehely 2005).

Generell planen die Kommunen derzeit, den Anteil vollzeittätiger Pflegekräfte zu erhöhen, wobei dies mit der schwierigen ökonomischen Situation Teilzeitbeschäftigter und der Notwendigkeit, langfristig Pflege und Versorgung in einer alternden Gesellschaft sicherzustellen, begründet 
wird. Dies setzt jedoch eine Anpassung der Arbeitsbelastung an eine Vollzeittätigkeit voraus, denn vollzeittätige Pflegehelferinnen und -helfer fühlten sich physisch und psychisch deutlich erschöpfter und belasteter als Teilzeitkräfte. Teilzeittätigkeit kann folglich auch als individuelle Strategie zur Reduktion einer starken Arbeitsbelastung interpretiert werden (Gustafsson/Szebehely 2005).

\section{AKTUELLE PERSONALPOLITIK}

Seit Ende der 1990er Jahre wird wieder vermehrt Personal eingestellt. Dennoch kann die Personalaufstockung die ansteigenden Aufgaben in der Pflege noch nicht kompensieren (Szebehely 2000). Trotzdem zeigt ein Vergleich der Ergebnisse einer Befragung von Pflegehelferinnen aus dem Jahr 2000 und 2003 eine Verbesserung der Arbeitssituation, wobei allerdings die Belastungswerte immer noch über den Werten zu Anfang der 1990er Jahre liegen (Gustafsson/Szebehely 2005). Der Einfluss der Zulassung privater Dienstleister seit den 1990er Jahren auf die Arbeitssituation ist nur schwierig einzuschätzen, denn es liegen nur wenige Forschungsergebnisse dazu vor. Einige Befunde weisen darauf hin, dass sich die kommunalen, öffentlichen Anbieter aufgrund der Konkurrenzsituation den privaten Anbietern annähern. Als entscheidend für die Arbeitssituation erweisen sich jedoch die ökonomischen Ressourcen, die zur Verfügung stehen, und nicht die Trägerschaft an sich (Trydegard 2005). Auch eine direkte Vergleichsuntersuchung aus dem Jahr 2003 fand kaum Unterschiede in der Arbeitssituation bei Pflegekräften in der häuslichen Pflege in privater oder kommunaler Trägerschaft, während sich deutliche Unterschiede zwischen den Kommunen abzeichneten (Gustafsson/Szebehely 2007).

Im Mittelpunkt der derzeitigen Personalpolitik steht die ausreichende Rekrutierung von Pflegekräften, deren adäquate Qualifizierung und die Verbesserung der Arbeitssituation, um den zukünftigen Bedarf an qualifizierten Pflegekräften zu sichern (Trydegard 2005). Seit den 1990er Jahren werden dafür vermehrt in Schweden lebende Pflegekräfte mit einem Migrationshintergrund eingestellt. Ihr Anteil unter den Neueinstellungen betrug $200520 \%$ bei einem Anteil von $13 \%$ an den Pflegekräften insgesamt. Die Altenbetreuung weist nach wie vor die gleiche hierarchi- sche Berufsstruktur wie Ende der 1980er Jahre auf: Krankenschwestern in Schweden, die über einen Hochschulabschluss verfügen, organisieren und leiten die Versorgung, während Pflegehelferinnen und -helfer mit einer mittlerweile dreijährigen Berufsausbildung die alltägliche Versorgung durchführen (Übersicht 1). 2005 hatten $56 \%$ aller Pflegehilfskräfte eine Ausbildung in der Pflege abgeschlossen, während die weiteren zumeist eine nicht-pflegebezogene Berufsausbildung aufwiesen. Die Vermittlung einer adäquaten Ausbildung in der Pflege für die ca. $40 \%$ der Pflegekräfte ohne formale Pflegeausbildung wird mit unterschiedlichen Strategien verfolgt. Dazu gehört beispielsweise die Entwicklung von Ansätzen zur Bewertung und Anerkennung von im Berufsalltag gewonnenen Kompetenzen (SOS 2002). Unterstützt werden die Anstrengungen auf kommunaler Ebene in den Jahren 2005-2007 durch ein nationales Förderungsprogramm (Sveriges Kommuner och Landsting 2006).

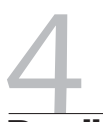

\section{Resümee: Altenbetreuung als Beruf - Möglichkeiten und Grenzen}

Die Vergesellschaftung von Fürsorgearbeit für ältere Menschen unter öffentlicher Regie in Schweden führte zum Aufbau eines Arbeitsmarktsegments und ermöglichte so Frauen mit einer einfachen oder ohne formale Qualifikation einen Zugang zu regulärer Erwerbsarbeit. Pflegetätigkeiten, die in Schweden zur regulären Erwerbsarbeit wurden, werden in Deutschland - teilweise illegal - über einen grauen Markt hergestellt oder eben nur symbolisch bezahlt innerhalb der Familie ausgeführt. Offensichtlich wird: Je nachdem, wie Pflegearbeit organisiert wird, beeinflusst sie nicht nur die Ungleichheit zwischen Männern und Frauen in der Frage der Erwerbsbeteiligung, sondern auch Ungleichheiten zwischen Frauen unterschiedlicher Klassenzugehörigkeit und, wie neuere Entwicklungen zeigen, auch nach Ethnizität. Mittlerweile werden in Schweden lebende Migrantinnen und Migranten auch aus Ländern außerhalb der EU vermehrt als reguläre Pflegekräfte eingesetzt. Dabei handelt es sich häufig um Migranten, die schon als Flüchtlinge nach Schweden kamen.
Die Analyse des schwedischen Beispiels zeigt auch, dass sich Pflegearbeit erst allmählich als eine Tätigkeit mit regulären Anstellungsformen, Arbeitszeiten und Qualifikationsanforderungen herausgebildet hat. Die positive Entwicklung wurde durch die Restriktion ökonomischer Ressourcen zu Beginn der 1990er Jahre unterbrochen und zum Teil zurückgedreht. Die seit Ende der 1990er Jahre wieder verstärkte Bereitstellung ökonomischer Ressourcen und die zunehmende Einstellung von Pflegekräften konnten die negative Entwicklung zumindest begrenzen und teilweise positiv wenden. Die Ergebnisse machen deutlich, dass nicht die Frage öffentlicher oder privater Trägerschaft entscheidend für die Entwicklung im Berufsfeld war, sondern dass die öffentliche bzw. kollektive Bereitstellung ausreichender ökonomischer Ressourcen weit bedeutsamer ist.

Insbesondere seit den 1980er Jahren ist ein weiblich dominiertes Berufsfeld mit unterschiedlich qualifizierten Beschäftigten entstanden. Es entwickeln sich Lohnstrukturen, die mit anderen, jedoch ähnlich strukturierten Arbeitsmarktsegmenten vergleichbar sind. Durchgesetzt hat sich weiterhin die Einsicht, dass es beruflicher Kompetenzen bedarf, um Pflegetätigkeiten auszuführen. Dies spiegelt sich in den enormen Anstrengungen der Kommunen wider, den Pflegekräften, die zum Teil ohne Pflegeausbildung eingestellt werden, einen entsprechenden Zugang zu Ausbildungen zu verschaffen. Die Professionalisierung pflegerischer Tätigkeiten, ihre Organisation als reguläre Beschäftigung sowie die Schaffung einer mit anderen Arbeitsmarktsegmenten vergleichbaren Arbeitssituation machen solche Anstrengungen wie auch die Bereitschaft, in weiten Zeithorizonten zu denken, erforderlich. Die besonderen physischen und psychischen Belastungen sowie die geforderte zeitliche Flexibilität, wie z. B. durch Wochenendarbeit oder Nachtarbeit, stellen besondere Anforderungen an die Arbeitsorganisation von Pflegetätigkeiten. Zu einer entscheidenden Rahmenbedingung wird die kollektive Bereitstellung ausreichender ökonomischer Ressourcen für die Ausführung der Pflegetätigkeit. Der Aufbau des Berufsfelds hat diese besonderen Anforderungen, die damit verbundenen Schwierigkeiten, aber auch die Chancen für Frauen aufgrund eines Zugangs zum regulären Arbeitsmarkt erkennen lassen. 
Dass schwierige Arbeitsbedingungen und Arbeitsbelastungen ein länderübergreifendes Kennzeichen von Pflegetätigkeiten sind, zeigen international vergleichende Studien (Dellve 2003). Ein öffentlich finanzierter Arbeitsmarkt bietet Ansatzpunkte, ein vergleichbares, reguläres Arbeitsmarktsegment zu entwickeln sowie herauszustreichen, wie bedeutsam Kompetenz für die Ausübung der Tätigkeit ist. Dies wird deutlich in den Anstrengungen und Debatten zur Qualifizierung der Beschäftigten und zur Verbesserung ihrer Arbeitssituation in Schweden. Dagegen finden in Deutschland ähnlich schwierige Ar- beitsbedingungen der Beschäftigten, die durch ökonomische Restriktionen im Rahmen der Pflegeversicherungen noch verstärkt werden, nur seltener Beachtung. Deutlich ist auch geworden, dass die Anstellung von - zumeist - Frauen mit einer geringen oder keiner formalen Qualifikation nicht unbedingt als geeigneter Ausgangspunkt für die Entwicklung von Qualifizierungsmaßnahmen gelten kann. Eine solche Personalpolitik ist im Gegenteil eher dem Motiv der Kostenersparnis geschuldet. Allerdings müssen in diesem Zusammenhang auch die sehr unterschiedlichen Qualifikations- und Berufsstrukturen in beiden
Ländern in dem Tätigkeitsbereich beachtet werden. In Deutschland wird der Tätigkeitsbereich dominiert durch Pflegekräfte mit einer qualifizierten Berufsausbildung in der Krankenpflege oder der Altenbetreuung, während in Schweden Krankenpflegekräfte - immer mit Hochschulabschluss - Führungsaufgaben übernehmen und die Pflegehelferinnen und -helfer die alltägliche Betreuung ausführen. Trotz der Unterschiede belegen die schwedischen Erfahrungen, wie auch im Berufsalltag Qualifikationen vermittelt sowie Weiterbildung und Berufserfahrung als Qualifizierungsnachweis anerkannt werden können.

\section{LITERATUR}

\begin{abstract}
Äldreuppdraget (1996): Konkurrensutsättning och entreprenader i äldreomsorgen, Stockholm

Äldreuppdraget (2000): Slutrapport. Socialstyrelsen, Stockholm Anttonen, A. /Sipilä, J. (1996): European Social Care Services: is it possible to identify models, in: Journal of European Social Policy 2, S. 87-100 Anttonen, A./ Baldock, J./ Sipilä, J. (eds.) (2003): The Young, the Old and the State. Social Care Systems in Five Industrial Nations, Cheltenham Bäckman, O. (2001): Med välfärdsstaaten som arbetsgivare, in: SOU 52, Välfärdstjänster i omvandling. Socialdepartementet, Stockholm, S. 189238
\end{abstract}

Bettio, F./Plangena, J. (2004): Comparing care regimes in Europe, in: Feminist Economics 1, S. 85-113

Brodin, H. (2005): Does anybody care? Public and Private Responsibilities in Swedish Eldercare 1940-2000, Doctoral thesis, Umea

Burau, V./ Theobald, H./Blank, R. H. (2007): Governing Home Care: A Cross-National Comparison, Cheltenham

Daly, M./Lewis, J. (1998): Introduction: Conceptualising Social Care in the Context of Welfare State Restructuring, in: Lewis, J. (Hrsg.): Gender, Social Care and Welfare State Restructuring in Europe, Aldershot, S. 1-24 Dellve, L. (2003). Explaining occupational disorders and work ability among home care workers. (Akad.avh) Göteborgs universitet, Sahlgrenska akademin, Instiutionen för arbetsmedicin Esping-Andersen, G. (1990): The Three Worlds of Welfare Capitalism, Cambridge

Evers, A./Wintersberger, H. (Hrsg.) (1990): Shifts in the Welfare Mix, Frankfurt/Main

Evers, A./Svetlik, I. (Hrsg.) (1993): Balancing Pluralism. New Welfare Mixes in the Care for the Elderly, Aldershot

Evertson, L. (2000): The Swedish Welfare State and the Emergence of Female Welfare State Occupations, in: Gender, Work and Organization 4, S. 230-241

Graham. H. (1991): The concept of caring in feminist research. The case of domestic service, in: Sociology 25, S. 61-78
Gonäs, L./Johansson S./Svärd, I. (1997): Lokala utfall av den offentliga sektorns omvandling, in: Sundin, E. (Hrsg.) Om makt och kön. SOU 1997:83, Stockholm, S. 103-146

Gough, R. (1994): Fran hembiträden till social hemtjänst, in: Baude, A./ Runnström, C. (Hrsg.): Kvinnans plats i det tidiga välfärdssamhället, Stockholm, S. 39-56

Gustafsson, R. A./Szebehely, M. (2005): Arbetsvillkor och styrning i äldreomsorgens hierarki. Rapport i socialt arbete 114. Stockholms Universitet, Socialhögskolan, Stockholm

Gustafsson, R. A./Szebehely. M. (2007): Privat och offentlig äldreomsorg - svenska omsorgsarbetares syn pa arbetsmiljö och politisk styrning, in: Socialvetenskaplig Tidskrift 1, S. 47-66

Hirdman, Y. (1990): Genussystemet, in: SOU 44. Demokrati och Makt I Sverige. Makturedningens huvudrapport, Stockholm, S. 73-116 Hochschild, A. R. (2000): The nanny chain, The American Prospect 4, S. 32-36

Johansson, S. (1997): Hälsoprofessioner i välfärdstatens omvandling, in: Sundin, E. (Hrsg.) Om makt och kön. SOU 83, Stockholm, S. 69-102 Johansson, S. /Noren, P. (2001): National Report, Sweden WP3 Mapping of Care Services and the Care Workforce. Report: EU-Project Care Work in Europe. Current Understandings and Future Directions

Knijn, T./Kremer, M (1997): Gender and the caring dimensions of welfare states, in: Social Politics, Fall, S. 328-361

Lamura, G. (forthcoming August 2007): Italy's Familistic Approach to Elderly Care and the New Role of Migrant Home Care Workers, in: Burau, V./Theobald, H./Blank, R. H. (Hrsg.): Governing Home Care: A CrossNational Comparison, Cheltenham, S. 118-121

SCB (1990): Unveröffentlichte Daten zum sozialen Dienstleistungsbereich, Stockholm

SCB (1993): Löner I Sverige 1990-1991, Stockholm

SCB (2007): Arbetskraften efter kön 2000-2007. Stockholm (www.scb.se) download 21.6.2007 
SOS (2002): Kompetenskrav för personal inom vard och omsorg om äldre. Socialstyrelsen, Stockholm

SOS (2004): Konkurrensutsättning och entreprenader inom äldreomsorgen. Utvecklingsläget 2003, Stockholm

Statistisches Bundesamt (2007a): Pflegestatistik 2005. Deutschlandergebnisse, Wiesbaden (www.destatis.det) download 21.6.2007

Statistisches Bundesamt (2007b): Statistik zur Erwerbsbeteiligung, Wiesbaden (www.destatis.de) download 25.6.2007

Sveriges Kommuner och Landsting (2006): Aktuellt om äldreomsorgen November 2006, Stockholm

Szebehely, M. (1995): Vardagens Organisering, Lund

Szebehely, M. (1996): Hemhjälp under skiftande villkor - om gamla och hjälpgivare I den svenska hemtjänsten. Särtryckserien, Stockholm

Szebehely, M. (1998): Changing Divisions of CareWork: Caring for Children and Frail Elderly People in Sweden, in: Lewis, J. (Hrsg.): Gender, Social Care and Welfare State Restructuring in Europe, Aldershot, S. 257-283
Szebehely, M. (2000): Äldreomsorg i förändring - knappare ressurser och nya organisationsformer, in: SOU (2000:38): Välfärd, vard och omsorg Antologi/Kommitten Välfärdsbokslut, Stockholm, S. 171-223

Theobald, H. (1999): Geschlecht, Qualifikation und Wohlfahrtsstaat. Deutschland und Schweden im Vergleich, Berlin

Theobald, H./Maier, F. (2002): Women between Labour Market Integration and Segregation. Germany and Sweden Compared, in: Mosley, H./O`Reilly, J./Schömann, K. (Hrsg.): Labour Markets, Gender and Institutional Change. Essays in Honour of Günther Schmid, Cheltenham, S. 212-241

Theobald, H. (2004): Care services for the elderly in Germany, Infrastructure, access and utilisation from the perspective of different user groups. (SP I 2004-302). Discussion paper. Working Group Public Health Policy. Social Science Research Center (WZB), Berlin

Trydegard, G. B. (2005): Äldreomsorgspersonalens arbetsvillkor i Norden - en forskningsöversikt, in: Szebehely, M. (Hrsg.): Äldreomsorgsforskning in Norden, Köpenhamn, S. 143-195 